\title{
New twists for an old turning illusion
}

\author{
Mark Chappell, Johanne L Hawke \\ School of Applied Psychology (MG) and Applied Cognitive Neuroscience Unit, Griffith Health Institute, \\ Griffith University, Nathan, QLD 4111, Australia; e-mail: m.chappell@griffith.edu.au \\ Received 25 August 2011, in revised form 23 September 2011
}

\begin{abstract}
A Vernier-offset illusion induced by rotating lines, introduced by Matin et al (1976 Perception \& Psychophysics 20138 -142) was re-examined using onset, offset, and reverse trajectories inspired by flash-lag illusion research, with both Vernier and alignment-with-vertical judgments being recorded. The pattern of illusions found was generally in agreement with a differential latency of stimulus ends account described by those authors, although certain variants of modern spatial projection theories, and a differential latency of attribute account, could also accommodate much of the data.
\end{abstract}

Matin et al (1976) reported that two lines rotating about a central point are perceived to have an illusory Vernier offset-see figure 1.

We replicated their finding (white lines were $2 \mathrm{deg} \times 0.05 \mathrm{deg}$, centres $3 \mathrm{deg}$ from the central fixation, rotating at $0.66 \mathrm{~Hz}, 50 \%$ clockwise), but supplemented their main condition, with lines rotating continuously, with trajectories inspired by flash-lag research (Eagleman and Sejnowski 2007; Nijhawan 2008). In these, the lines either appeared suddenly and rotated (onset trajectory), or after some time they suddenly disappeared (offset trajectory), or the lines suddenly reversed their direction of rotation. For each of these latter trajectories our eleven subjects made a Vernier judgment at the defining event for the trajectory (when lines were vertical), and when they were vertical for the continuous trajectory (in a control condition the lines were vertical and stationary). In an adaptive method of constant stimuli, the Vernier offset was varied (by equal and opposite amounts for the upper and lower lines) and subjects indicated if the top line was to the left or the right of the bottom one.

In a separate set of orientation judgment conditions, subjects indicated if the lines were rotated to the left or right of vertical when the trajectory defining event occurred, for those trajectories which had one. For these, the lines were displayed radial from the fixation (as in figure 1a). We report this illusion as the distance of the centres of the stimuli (from vertical) along their trajectories, at which the events were perceived to occur, to facilitate comparison with the Vernier judgment data.

Logistic regression fits yielded PSEs for each subject condition, and figures 1c and 1d show the means of these. For the Vernier judgments, as well as finding a significant illusion for the continuous trajectory as Matin et al (1976) did, our subjects perceived the lines to be displaced forward, as in figure $1 \mathrm{~b}$, as they disappeared with the offset trajectory, and displaced forward in the direction of their motion before reversal with the reversal trajectory. As the lines suddenly appeared with the onset trajectory, their alignment was perceived veridically, on average (subjects often reported difficulty making this judgment at onset).

As one account of their data, Matin et al (1976) suggested that, because the stimulus energy was spread across a larger range of retina for the outer ends of the lines compared to the inner ends, the outer ends had a longer latency to perception (a differential latency account), and thus were perceived to trail. Our similar results with offset and reversal trajectories could be explained by Matin et al's (1976) account if the alignment 


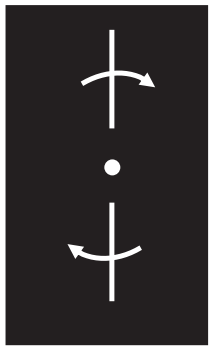

(a)

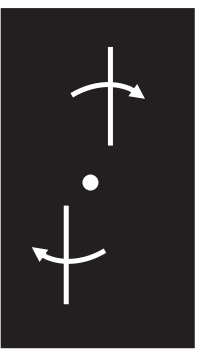

(b)

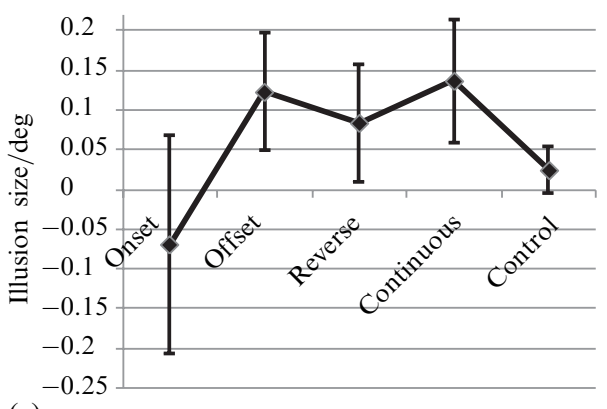

(c)

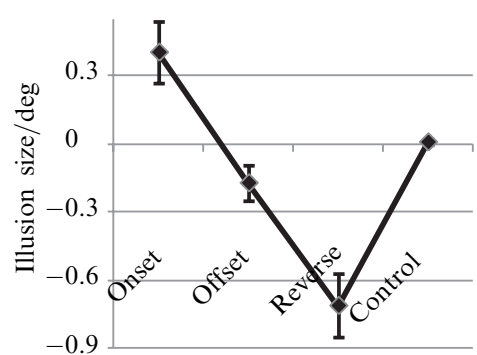

(d)

Figure 1. Our stimuli. (a) Displayed - two lines rotate around a fixation point. (b) Perceivedthey appear offset in their direction of motion. (c) Our Vernier and (d) orientation data with eleven subjects. Error bars are 95\% confidence intervals.

could be reported when the inner ends were perceived to reach the extreme of their trajectory, and before the outer ends did (so they would not appear vertical, although they were). In agreement with this account, with orientation judgments for the offset and reversal trajectories this is just what was reported: the lines were not perceived to reach the vertical orientation. We note, however, that this 'undershoot' result with the orientation offset judgment is at odds with most findings with this trajectory, which find no illusion (eg Chappell et al 2006), although both undershoots (eg Maus and Nijhawan 2009), and overshoots (eg Maus and Nijhawan 2006) have been reportedsee below for a possible account of our undershoot.

On the face of it, our observed forward displacements of the lines with Vernier judgments could provide support for spatial projection theories of motion perception (Eagleman and Sejnowski 2007; Nijhawan 2008). However, note that if parts of the line were projected forwards by distances in proportion to their speed, which could give the closest approach to veridical perception, then no Vernier illusion would be predicted. In order to account for this illusion, these theories would have to posit a projection tangential to the motion, but with no (or little) 'twisting' of the stimuli. In that case, to predict our data, motion before the trajectory defining event would have to be the determinant of the direction of projection, in agreement with Nijhawan (1994), but not with Eagleman and Sejnowski (2007) [also cf Matin et al's (1976) discussion re figural induction effects]. However, Nijhawan (2008) has supplemented his theory to suppose that such events terminate the projection, so that it could not now account for our Vernier data with an offset trajectory. Our orientation results with onset and reversal trajectories do support the latest versions of the spatial projection theories (Eagleman and Sejnowski 2007; Nijhawan 2008).

Could orientation of our rotating stimuli be processed as a separate attribute (cf colour-Kreegipuu and Allik 2004), with a greater latency than the computation of position-a differential latency of attribute account? One would have to further suppose that the full perception of the stimulus is reconstructed using just the computed position 
of the centre, and computed orientation, of the stimulus. In this case sensory signals might cause the object's disappearance before its final orientation was computed, accounting for our non-null orientation offset result. For other conditions, this is similar to Matin et al's (1976) differential latency account, and results are predicted similarly.

Our account might seem to predict a Vernier illusion at onset, which we did not find. However, it may be that the processes responsible for the latency advantage that position perception enjoys take some time to be set up (cf Öğmen et al 2004), so that there is initially no latency advantage over orientation.

Matin et al's (1976) illusion provides another useful test of theories of the perception of the position of moving objects, albeit with some interpretational twists and turns.

Acknowledgments. We would like to thank the Applied Cognitive Neuroscience Unit for support. This work was the basis of an Honours thesis project for the second author.

\section{References}

Chappell M, Hine T J, Acworth C, Hardwick D, 2006 "Attention 'capture' by the flash-lag flash" Vision Research $463205-3213$

Eagleman D M, Sejnowski T J, 2007 "Motion signals bias localization judgments: A unified explanation for the flash-lag, flash-drag, flash-jump, and Frohlich illusions" Journal of Vision 7(4):3 $1-12$

Kreegipuu K, Allik J, 2004 "Confusion of space and time in the flash-lag effect" Perception 33 $293-306$

Matin L, Boff K H, Pola J, 1976 "Vernier offset produced by rotary target motion" Perception \& Psychophysics $20138-142$

Maus G W, Nijhawan R, 2006 "Forward displacements of fading objects in motion: The role of transient signals in perceived position" Vision Research $464375-4381$

Maus G W, Nijhawan R, 2009 "Going, going, gone: Localizing abrupt offsets of moving objects" Journal of Experimental Psychology: Human Perception and Performance 35 611-626

Nijhawan R, 1994 "Motion extrapolation in catching" Nature 370 256-257

Nijhawan R, 2008 "Visual prediction: Psychophysics and neurophysiology of compensation for time delays" Behavioral and Brain Sciences 31 179-239

Öğmen H, Patel S S, Bedell H E, Camuz K, 2004 "Differential latencies and the dynamics of the position computation process for moving targets, assessed with the flash-lag effect" Vision Research $442109-2128$ 


\section{PERCEPTION}

VOLUME 412012

www.perceptionweb.com

Conditions of use. This article may be downloaded from the Perception website for personal research by members of subscribing organisations. Authors are entitled to distribute their own article (in printed form or by e-mail) to up to 50 people. This PDF may not be placed on any website (or other online distribution system) without permission of the publisher. 